\title{
A Preliminary Validation of the Polish Version of the Social Media Fatigue Scale
}

\author{
Agata H Świątek' \\ Małgorzata Szcześniak (D) \\ Shiyi Zhang ${ }^{2}$ \\ Hanna Borkowska' \\ 'Institute of Psychology, University of \\ Szczecin, Szczecin, 7I-017, Poland; \\ ${ }^{2}$ Collaborative Innovation Center of \\ Assessment for Basic Education Quality, \\ Beijing Normal University, Beijing, \\ People's Republic of China
}

Background: As social media fatigue is a newly described phenomenon, we do not possess many tools to measure this concept. The main aim of the study was to establish a latent structure of the Social Media Fatigue Scale (SMFS) and to identify whether the Polish version is congruent with the Chinese version. The second aim was to examine whether the factor structure fits the data and yields a comparable goodness-of-fit index. The third aim was to consider the convergent validity of the SMFS and to verify whether tiredness and Internet addiction correlate positively.

Methods: The research was conducted on a group of 331 (1st Study) and 379 (2nd Study) adolescents and young adults. It was performed using the SMFS and Internet Addiction Test (IAT).

Results: The outcomes suggest that the three-factor structure is the optimal and reliable solution that corresponds to the original SMFS. CFA provided a good fit. All the dimensions of the SMFS correlated positively with Internet addiction.

Conclusion: The Polish version of the SMFS presented satisfactory psychometric properties, showing many similarities with the original Chinese version of the SMFS. The findings confirm that excessive use of social media may lead to discomfort and negative emotions related to feeling overwhelmed by information, social interaction overload, and personal/ others' expectations.

Keywords: social media fatigue, Internet addiction, adolescents, young adults

\section{Introduction}

In January 2021, the Global Overview Report presented information about the total size of the global social network population. According to its data, active social media users have reached 4.20 billion, which is almost $54 \%$ of the entire world population. Compared to the previous year, the number of people using social networking sites has grown by more than $10 \%{ }^{1}$ In Poland, $99 \%$ of adolescents and 98\% young adults were the two largest groups of Internet users in 2020. ${ }^{2}$ These figures confirm that social media usage has become omnipresent in the world at large, ${ }^{3}$ playing an essential function in people's lives. ${ }^{4}$ On the other hand, some statistics reveal that $42 \%$ of Facebook users in the United States have taken a break from checking the site for several weeks or more and $26 \%$ have removed the app from their mobile phones. ${ }^{5}$ Moreover, several different studies showed that some users of well-known social media platforms are considering temporary disconnection from Instagram, ${ }^{6,7}$ Facebook, ${ }^{6,8}$ WeChat, ${ }^{9}$ MySpace, ${ }^{10}$ WhatsApp, ${ }^{11}$ or Twitter. ${ }^{12}$
Correspondence: Małgorzata Szcześniak Email malgorzata.szczesniak@usz.edu.pl 


\section{Literature Review}

\section{Social Media Fatigue}

There is no consensus on a concept that captures the meaning of declining interest within the number of active users. ${ }^{4}$ Although many authors define this phenomenon differently, using terms such as "social network fatigue," "10,13,14 "social networking services fatigue,"12,15 "social media burnout,", 16 and "social media fatigue,",3,5,11,17-19 all of them adhere to the same type of trend related to quitting or abandoning social networking services. Given that social media fatigue (hereinafter: SMF) seems to be the most popular and common term in the scientific publications, we decided to assume this concept in the present research.

Prior empirical investigations have determined various antecedents of leaving social media. ${ }^{18}$ Building on the current literature, Hong and $\mathrm{Oh}^{8}$ listed eight more prevalent reasons for social media withdrawal: the concern for privacy, the appearance of new competitive platforms, peer or family pressure, triviality/banality/irrelevance, annoying or deceptive content, information overload, fear of addiction, and wasting time. Moreover, other authors ${ }^{15,20,21}$ reported SMF as a somehow less apparent, but equally important, reason for the decline in active user numbers.

The specific type of fatigue that results from spending time on social media is a relatively recent topic in research on human activity on the Internet. ${ }^{13,18,22}$ The definitions of the phenomenon of SMF vary in several sources since fatigue is a complex concept. ${ }^{4,21}$ In a broad sense, the term of SMF refers to a personally experienced feeling of tiredness connected to the use of social media applications. ${ }^{13,19-21}$ In their study, Ravindran et al (p. 2317) ${ }^{13}$ described SMF as

a subjective, multidimensional user experience comprising feelings such as tiredness, annoyance, anger, disappointment, guardedness, loss of interest, or reduced need/motivation associated with various aspects of social network use and interactions.

Bright and colleagues ${ }^{17,23}$ defined SMF as a user's tendency to retreat from social network engagement when they are overloaded with too much information. Other studies showed that SMF refers to suffering from mental $^{12,18}$ or emotional ${ }^{16,24}$ exhaustion, and manifests itself in decreased interest in accessing to social platforms. ${ }^{4,10}$

The presented definitions confirm that SMF reflects a multidimensional user experience ${ }^{5}$ in cognitive, behavioral, and emotional dimensions. In terms of the cognitive aspect, SMF encompasses perceived information avoidance $^{10,25}$ that generally emerges when users are exposed to a quantity of information ${ }^{13,20,26,27,28}$ in excess of what they can receive and process. Indeed, some studies furnished empirical evidence of the positive association between information overload and $\mathrm{SMF}^{20}$ Likewise, Zhang et $\mathrm{al}^{5}$ found that not only the amount of information has an impact on fatigue in relation to social media, but also its complexity, misinformation, disinformation, and mal-information.

With respect to the behavioral dimension, information overload may enhance the potentially adverse behavior of users toward different social media platforms. For example, Guo et al ${ }^{10}$ observe that too much information may lead to omitting or bypassing less significant facts, or even stopping using social network services. Moreover, the behavioral expression of SMF may refer to actions that originally were not intended by media users. When people start "drifting" among the content that scrolls by on the screen and continue to click messages that appear at the moment, they may fail to remember why they logged into their social networking accounts. According to cognitive psychology, such forgetfulness may result from the fact that only some information is stored in long-term memory ${ }^{29}$ and probably most of our social media activity does not go beyond episodic and short-term memory. Likewise, forgetting may be related to the passage of time $^{30}$ because the longer we stop browsing other content on social media, the less chance we have to remember why we logged on.

Regarding the emotional component of SMF, social media users sometimes experience ambivalent or negative emotions. For example, some authors ${ }^{9,31}$ point out that processing information and wasting time are associated with an inner sense of guilt and self-accusation. A growing body of literature qualifies anxiety as a negative outcome of social media fatigue as well. ${ }^{18,32,33}$ Among other emotional components of SMF, boredom, ${ }^{34}$ decline in interest, ${ }^{34}$ irritation, ${ }^{17,23}$ and frustration $^{23}$ are also mentioned.

\section{Measuring SMF}

As SMF is a newly described phenomenon, we do not possess many tools to measure this concept. The research review shows that the first attempts to define SMF were associated with the presentation of several free items or one-factor scales based on theoretical literature or adapted 
from social media market research. For example, Maier et $\mathrm{al}^{35}$ developed four separate tools assessing: social overload (eg: "I feel irritated, because I pay too much attention to posts of my facebook-friends in Facebook"), strain (eg: "It is hard for me to relax after using Facebook"), satisfaction ("Overall, I am very satisfied with Facebook"), and discontinuous usage intention (eg: "In future, I will use Facebook far fewer than today"). All of the scales measure their constructs on a 7-point Likert scale. Next, Bright et $\mathrm{al}^{17}$ created the "Social Media Fatigue" questionnaire, based mainly on research by Gartner. The tool consisted of 5 statements relating primarily to two reasons for being tired: too much information and not enough detail on social media sites. Likewise, Dhir and colleagues ( $p$. 146) ${ }^{18}$ computed SMF using three items: "I am likely to receive too much information when I am searching on FB"; "I am frequently overwhelmed by amount of information available on FB"; "Amount of information available on FB makes me tense \& overwhelmed". Finally, Lee et $\mathrm{al}^{36}$ created a questionnaire called the "Social Media Fatigue Scale." This multidimensional tool, with a structure resembling the Maslach Burnout InventoryHuman Services Survey, evaluates the degree of fatigue among social networking service users, assessing emotional exhaustion, depersonalization, lack of personal accomplishment, and privacy exposure risk.

Although, all presented SMF measures possess good or excellent reliability, which makes them applicable tools for estimating social media exhaustion, we opted for a validation of the Social Media Fatigue Scale (hereinafter: SMFS) developed and validated by Zhang et al. ${ }^{5}$ There are several reasons behind this choice. First of all, the previous scales lack information about the other remaining psychometric properties, such as validity, model fit, and explanatory power, which undoubtedly hinders the process of their use. Secondly, individual scales often relate to a specific situation or the application of a selected social networking platform (eg: Facebook). Moreover, the existing measurement scales most often include a single, selected perspective of understanding SMF (for example, only cognitive). Instead, the "Social Media Fatigue Scale," being a multidimensional measure of social media tiredness, includes its three dimensions: cognitive, behavioral, and emotional.

The authors of the scale ${ }^{5}$ built their tool referring to the Limited Capacity Model of Mediated Message Processing ${ }^{37,38}$ which assumes that people have limited cognitive resources of perceiving, encoding, comprehending, and recalling the information they receive. Thus, when only reduced reserves are accessible, processing declines and individuals become fatigued. According to Zhang et $\mathrm{al}^{5}{ }^{5}$ this model not only permits an understanding of SMF in the context of the cognitive dimension, but also helps to explain it in the behavioral and emotional aspects. Following this approach, cognitive information overload can coincide with a change of behavior, which manifests itself, for example, in the form of forgetting the activities that were supposed to be performed or lacking the concentration (eg: "When I open a social media site, I may forget what I intended to post"). Being overwhelmed by the excess of messages provided by social media may lead to the decrease of activities on the media. Finally, the emotional dimension of SMF includes the unpleasant emotions associated with logging onto the account and receiving different notifications or invitations. Thus, overload and forgetfulness may cause a person's annoyment, irritation, anxiety, or fear that are part of media fatigue.

Since Zhang et $\mathrm{al}^{5}$ created a standardized 15-item scale to measure SMF that can be applied to any social platform, the main goal of Study 1 was to establish a latent structure of the SMFS and to identify via Exploratory Factor Analysis (EFA) whether the Polish version is congruent with the Chinese version of the measure. The aim of Study 2 was twofold. First, a Confirmatory Factor Analysis (CFA) was performed to examine whether the factor structure ascertained through EFA fits the data from the second sample and yields a comparable goodness-of-fit index as the original model. Second, the convergent validity of the SMFS was considered to verify whether social media fatigue is associated with Internet addiction. Both constructs seem to be connected with each other given that uncontrollable overuse of social media leads to high levels of different types of fatigue, independently of the users' age. ${ }^{39-41}$ Moreover, Duradoni et $\mathrm{al}^{42}$ report that social media addiction measures, both specific (related to overuse of Facebook) and general, correlate with positive (life satisfaction, vitality, happiness, flourishing, positive affect) and negative (negative affect) framed well-being. Therefore, based on the prior theoretical perspective ${ }^{42}$ and empirical evidence, ${ }^{43}$ which indicates that social networking site addiction and excessive involvement in using social media platforms may induce SMF, it was expected that media fatigue would positively correlate with excessive preoccupation with the Internet.

Unlike the original study by Zhang et al, ${ }^{5}$ which was conducted with the participation of adults, the current research was carried among both adolescents and young adults since engaging in various social media sites is one of their most frequent and intensive activities. ${ }^{44,45}$ A review of the literature 
shows that, in the majority of studies, the main focus has been on University students from Western, South or East Asian countries. ${ }^{18}$ To address the gap, the present research was to validate the structure of SMF in two quite large samples of Polish adolescents and young adults.

\section{Methods and Materials}

\section{Study I}

\section{Participants}

The research was conducted on a group of 331 adolescents and young adults (90\% women). The mean age of the respondents was $M=16.65$ with $S D=2.41$ (range $=14-25$ years). The participants were asked how long they had been using social media. More than half of them (56\%) replied with between 5 and 10 years, almost 33\% acknowledged less than 5 years, $10 \%$ declared more than 10 years, and only $1 \%$ less than a year. With respect to the use of social media sites which are popular in Poland, the following responses were given: 288 participants use Facebook; Instagram - 279; YouTube-272; TikTok-216; Snapchat-206; Pinterest-146; Twitter-60; WhatsApp - 32; LinkedIn - 4; ResearchGate -3 ; and Others -47 . When it comes to the declared time spent per day on social media, most participants indicated 3 or more hours $(67 \%)$, followed by those who use them for around 3 hours (19\%), 2 hours (9\%), and less than 1 hour (2\%). Only $3 \%$ of participants could not say how much time they spend online. When asked why they use social networking sites, 304 participants answered - entertainment (watching the lives of idols, funny videos, fashion and technical news); 279 - establishing and maintaining social contacts; 271 - performing professional/school duties; and 178 - improving competences (thematic groups, courses, training, and hobbies).

\section{Data Collection}

In the present study, the data were gathered via the online social networking among Polish speaking participants in March 2021. We used purposive sampling since adolescents and young adults, as a large group of social media users, were the target group of the present research. The underage participants took part in the study after obtaining their parents'/ guardians' consent. Adolescents were qualified to participate because the SMFS seems to be suitable for minors, who at this stage of life are developing great interest in social media. In fact, Internet use among teens and young adults has risen enormously in recent years. ${ }^{46,47,48}$ All participants were informed about the goal of the study, expressed their willingness to take part in it, and completed informed and written consent for their participation in the study. They were also guaranteed the confidentiality of their information. The study protocol was approved by the Bioethics Committee of the Institute of Psychology at the University of Szczecin (KB 2/ 2021) and performed in accordance with Declaration of Helsinki.

\section{Procedure of the Study}

We used the original version of the SMFS developed by Zhang et $\mathrm{al},{ }^{5}$ which consists of 15 statements and assesses three dimensions of SMF: cognitive, behavioral, and emotional. In the Instructions part, the respondents were asked to familiarize themselves with all the statements and indicate the degree to which they agree with each of them. All the items were rated on a 7-point Likert scale with $1=$ totally disagree; 2 = disagree; $3=$ partially disagree; $4=$ neither agree nor disagree; $5=$ partially agree; $6=$ agree; $7=$ totally agree.

In the process of validation, we implemented the forwardtranslation procedure. In the first step, a team of three independent linguists translated the SMFS from Chinese to Polish and two others from English to Polish. Next, two psychologists evaluated all facets of the translation and reached a consensus with respect to the items' sense or insufficiency. Subsequently, a group of thirty adolescents and students were asked to fill out the Polish version and assess whether the items were clearly stated and easy to comprehend. They were also invited to read the Introduction to the SMFS to capture some cultural differences in the usage of social media platforms. The respondents indicated that they did not have any account on the platforms Weibo, WeChat, RenRen, and Q-zone that are probably more well-known in China and East Asian countries. In place of the above-mentioned online services, we included those which are more popular in Poland: Facebook, Instagram, TikTok, Snapchat, YouTube, WhatsApp, and Pinterest. After that, bilingual translators without prior knowledge of the questionnaire did three back-translations which were found to be in agreement with the source version. The final Polish adaptation, together with the items of the original version of the SMFS, is accessible in the Appendix.

\section{Statistical Analysis}

The IBM SPSS Statistics 23.0 (Studies 1 and 2), AMOS with Maximum Likelihood Estimation version 21 (Study 2), and $\mathrm{G}^{*}$ Power software were used to perform the analyses. Before the other statistics, $G^{*}$ Power 3.1.9.4 ${ }^{49}$ was used to calculate the pertinent sample size. In the current research design, it was necessary to consider that the original sample would be split into two groups of respondents (one group for Study 1 and another group for Study 2). As the basic point for determining 


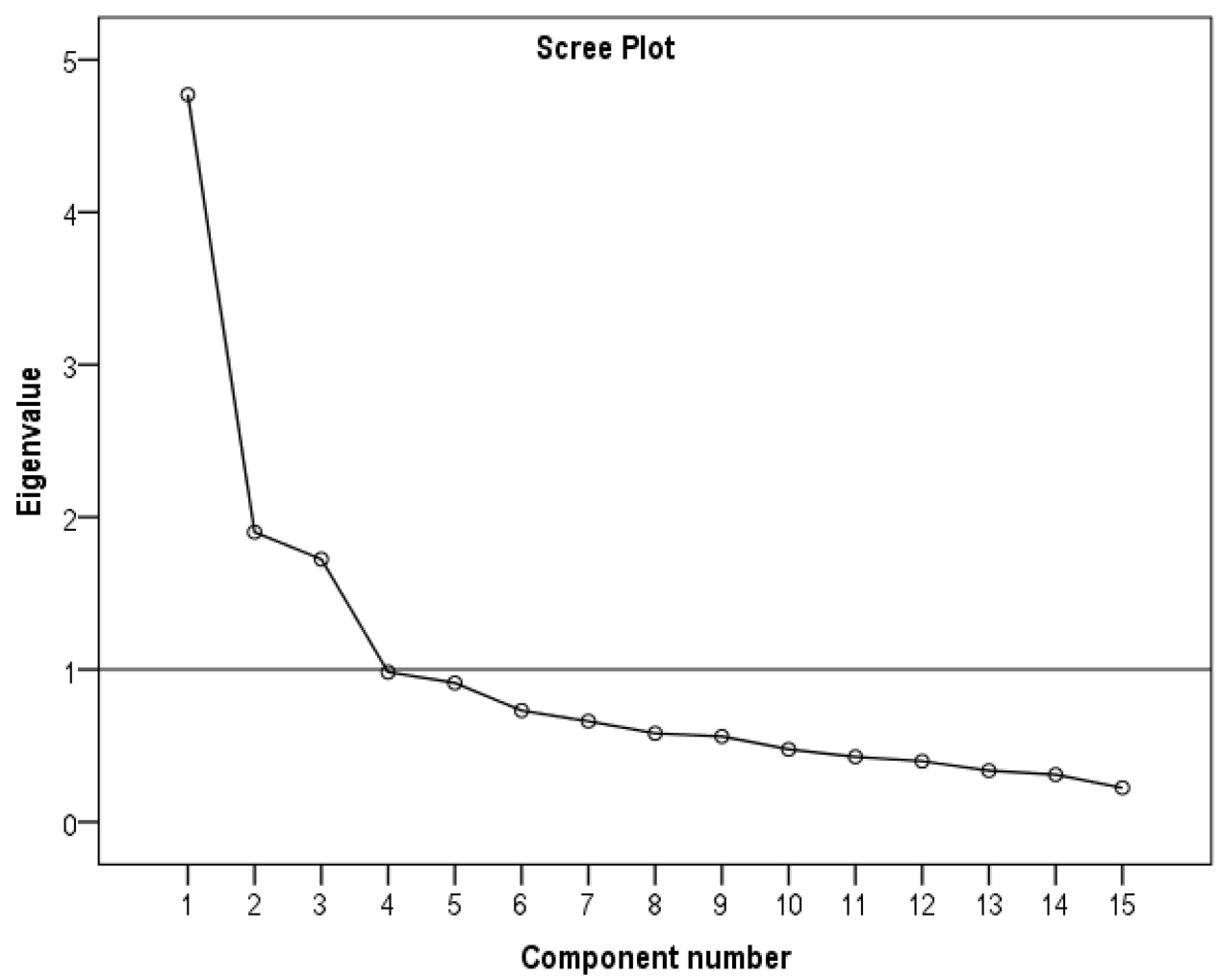

Figure I Scree plot of eigenvalues of the SMFS after principal component analysis. The number of components is on the $x$-axis and the eigenvalue on the $y$-axis.

the size of the whole group, we considered the empirical evidence regarding the correlation between SMF and Internet addiction (Study 2). We employed the higher power criteria of 0.95 and a significant criterion $\alpha$ of 0.05 to detect a realistic effect $(r=0.20)$. The rationale for using a rather small correlational value was because the existing studies examining the association between media fatigue and other variables related to social media addiction show rather contrasting results. For example, Whelan and colleagues ${ }^{34}$ report a correlation between SMF and social media use intensity equal to $r=$ 0.14 . In turn, Dhir et al ${ }^{18}$ observe that compulsive social network use and SMF fatigue are moderately associated $(r=$ $0.50)$. Moreover, the meta-analysis of 708 correlations deriving from the social and personality literature demonstrate a typical effect-size equivalent of $r=0.20 .^{50} \mathrm{G}^{*}$ Power suggested that we would need 319 respondents for group for Study 2 . Consequently, we assumed that the original sample would require at least 638 subjects.

Finally, to establish the factor structure of the Polish version of the SMFS, an exploratory factor analysis (EFA) was performed, using the principal components method with the oblique rotation method since, on the bases of the theory and original SMFS results, we expected the factors to be correlated. The discriminant power of the test items was estimated using the item-scale correlation coefficient. The analysis of the relationship between the results of a given item and the overall score of the scale was performed.

\section{Results}

\section{Exploratory Factor Analysis}

Based on the Kaiser criterion, the analysis allowed for the identification of three factors with eigenvalues higher than 1. They together explained $55.97 \%$ of the variance $(1 \mathrm{st}$ factor accounted for $31.81 \%$; 2nd - $12.67 \%$; 3rd $11.49 \%$ ). The KMO value was 0.825 , while Bartlett's Test of Sphericity test was found to be statistically significant: $\chi^{2}(105)=1773.63 ; p<0.001$, indicating the validity of the factor analysis. Drawing on the examination of the scree plot (Cattell's criterion, Figure 1), three factors could be distinguished.

The three-factor structure corresponds to the assumed theoretical and empirical model of the SMFS. The values of the factor loadings were at an adequate level, with the lowest value of 0.349 for the item smfs12, and the highest of 0.886 for the item smfs9 (Table 1). Although there are different opinions about the acceptable value of the factor loadings, we 
Table I Matrix of Rotated Components (Oblique Rotation with Kaiser Normalization) for the Items of the SMFS

\begin{tabular}{|l|c|c|c|}
\hline \multirow{2}{*}{ Items } & \multicolumn{3}{|c|}{ Factors } \\
\cline { 2 - 4 } & $\mathbf{I}$ & $\mathbf{2}$ & $\mathbf{3}$ \\
\hline Cronbach's $\alpha$ & 0.808 & 0.806 & 0.727 \\
\hline smfs9 & $\mathbf{0 . 8 8 6}$ & & \\
\hline smfs8 & $\mathbf{0 . 8 6 6}$ & & \\
\hline smfs7 & $\mathbf{0 . 7 9 3}$ & & \\
\hline smfs 10 & $\mathbf{0 . 6 2 2}$ & & \\
\hline smfs6 & $\mathbf{0 . 5 2 0}$ & & \\
\hline smfs4 & & $\mathbf{0 . 8 5 7}$ & \\
\hline smfs2 & & $\mathbf{0 . 7 6 9}$ & \\
\hline smfs3 & & $\mathbf{0 . 7 5 2}$ & \\
\hline smfs I & & $\mathbf{0 . 7 3 8}$ & \\
\hline smfs5 & & $\mathbf{0 . 5 9 8}$ & \\
\hline smfs 13 & & & $\mathbf{0 . 4 9 8}$ \\
\hline smfs 14 & & & $\mathbf{0 . 8 4 7}$ \\
\hline smfs 15 & & & \\
\hline smfsII & & & \\
\hline smfs 12 & & & \\
\hline I & & & \\
\hline
\end{tabular}

Notes: The items included in three factors are presented in bold. Values above 0.3 were established as inclusion criteria.

assumed a viewpoint of load of 0.30 to $0.40 .{ }^{51}$ In fact, some researchers ${ }^{52}$ observe that the threshold often used is to exclude overpass factor loadings which have absolute values lower than 0.30 . The results of the reliability analyses (Cronbach's alpha) confirm that three dimensions have sufficient reliability (Table 1), being above the traditional cut-off of 0.70 .

\section{Discriminant Power Analysis}

The performed analysis of the discriminant power (Table 2) of the items showed that all the items were positively correlated with the overall result of the test, which suggests that the higher the results obtained by the examined person for a particular item, the higher the results obtained for the remaining questions of the scale. All items indicate a weak and moderate level of discrimination, assuming coefficients in the range $0.361-0.578$.
Table 2 Discriminatory Power of Items on the SMFS

\begin{tabular}{|l|c|}
\hline Items & Item-Scale Correlation \\
\hline smfs I & 0.550 \\
\hline smfs2 & 0.503 \\
\hline smfs3 & 0.477 \\
\hline smfs4 & 0.438 \\
\hline smfs5 & 0.447 \\
\hline smfs6 & 0.461 \\
\hline smfs7 & 0.578 \\
\hline smfs8 & 0.537 \\
\hline smfs9 & 0.543 \\
\hline smfs 10 & 0.415 \\
\hline smfs II & 0.446 \\
\hline smfs 12 & 0.382 \\
\hline smfs 13 & 0.361 \\
\hline smfs 14 & 0.455 \\
\hline smfs 15 & 0.471 \\
\hline
\end{tabular}

\section{Study 2}

\section{Participants and Data Collection}

Study 2 was carried out with the participation of 379 adolescents and young adults (91\% women). The mean age of the respondents was $M=16.59$ with $S D=2.23$ (range $=14-25$ years). Since we used the split-sample method that allowed us a random division of the whole group into two halves, the procedure of data collection, statistical software, and sociodemographic variables were the same as those presented in Study 1. More than half of the participants (61\%) replied that they had been using social media between 5 and 10 years, almost $28 \%$ acknowledged less than 5 years, and $11 \%$ declared more than 10 years. With respect to the use of social media sites which are popular in Poland, the following responses were given: 347 participants use Facebook; Instagram - 326; YouTube - 307; TikTok - 257; Snapchat - 251; Pinterest 158; Twitter - 74; WhatsApp - 35; LinkedIn - 1; ResearchGate -2 ; and Others -54 . With respect to the declared time spent per day on social media, most participants indicated 3 or more hours (74\%), followed by those who use them for around 3 hours (15\%), 2 hours (6\%), and 
less than 1 hour $(0.3 \%)$. Only $4.7 \%$ of participants could not say how much time they spend online. When asked why they use social networking sites, 359 participants answered - entertainment (watching the lives of idols, funny videos, fashion and technical news); 337 - establishing and maintaining social contacts; 327 - performing professional/school duties; and 155 - improving competences (thematic groups, courses, training, and hobbies).

\section{Measurement}

Internet Addiction Test (IAT), created by Young and adapted into Polish by Hawi, Blachnio and Przepiorka, ${ }^{53}$ is a valid one-factor tool that measures Internet addiction. The instrument consists of 20 items (eg: "How often do you become defensive or secretive when anyone asks you what you do on-line?;" "How often do you fear that life without the Internet would be boring, empty, and joyless?"). Participants indicated their answers on a 5-point scale where 1 means rarely and 5 means always. A score of 80 or above denotes problematic Internet use. Consistent with the overall Cronbach's alpha computed from 11 studies through a meta-analysis of the reliability of IAT ${ }^{54}$ the reliability of the questionnaire in our study was equal to $\alpha=0.88$.

\section{Results of Confirmatory Factor Analysis}

Since a meaningful three-factor oblique structure of the SMFS in the EFA was obtained, the same three-factor model (cognitive, behavioral, and emotional dimensions) was specified, using CFA. The general threshold values of the most common fit indices were used to estimate the model: CMIN/DF less than 3; Goodness-of-Fit Index (GFI), Adjusted Goodness-of-Fit Index (AGFI), and Comparative Fit Index (CFI) more than $0.9 ;{ }^{55}$ Root Mean Square Errors of Approximation (RMSEA) less than or equal to 0.06; LO and HI between 0.05 and 0.08 ; PCLOSE higher than or equal to 0.05 and lower than or equal to 1; $\mathrm{CN} 0.05$ and $\mathrm{CN} 0.01$ higher than or equal to $200 .^{56}$ The analysis yielded a marginal fit of the model to the data $(\mathrm{CMIN} / \mathrm{DF}=4.58 ; \mathrm{GFI}=0.925 ; \mathrm{AGFI}=0.897$; $\mathrm{CFI}=0.912 ;$ RMSEA $=0.071 ;$ LO $90=0.064 ; \mathrm{HI} 90=$ $0.078 ;$ PCLOSE $=0.000 ;$ HOELTER $0.05=196$; HOELTER $0.01=0.215$ ). Although some fit indices presented acceptable or reasonable levels, some others showed a mediocre fit. Therefore, additional connections between error terms (Figure 2) were included in the model based on modification indices and on theoretical justification. After adding covariance paths, the indicators were found to present a good fit for the data $(\mathrm{CMIN} / \mathrm{DF}=2.58$; GFI $=0.961 ;$ AGFI $=0.944 ;$ CFI $=0.961 ;$ RMSEA $=$ 0.047 ; LO $90=0.040$; HI $90=0.055$; PCLOSE $=0.702$; HOELTER $0.05=347$; HOELTER $0.01=0.382$ ). The only exception was the Chi-square, presenting a statistically significant $p=0.000$ and thus showing a poor fit. However, in models with large samples, a significant Chi-square is likely to occur. ${ }^{57}$

The standardized factor loadings, shown in Figure 2, exceeded 0.4 , and ranged from 0.43 to 0.84 . The correlations between three subscales of the SMFS were as follows: cognitive and behavioral $r=0.435(p=0.001)$, cognitive and emotional $r=0.397(p=0.001)$, behavioral and cognitive $r=0.403(p=0.001)$. The values of the internal consistency obtained in the present study for cognitive $\left(\alpha_{\operatorname{cog}}=0.779\right)$, behavioral $\left(\alpha_{\mathrm{beh}}=0.811\right)$, and emotional dimensions $\left(\alpha_{\mathrm{emot}}=0.728\right)$ were considered acceptable. The 15 -item SMFS $\left(\alpha_{\mathrm{cmfs}}=0.849\right)$ exceeded the value of the original Chinese version of the SMFS.

\section{Results of Convergent Validity}

As shown in Table 3, convergent validity was assessed by measuring the correlation (Pearson correlation coefficient) between three subscales of the SMFS and IAT.

There was a significant positive correlation of Internet addiction with all dimensions of SMF, indicating that the more time users spend on the Internet, the more they declare information overload, forgetting the activities that were supposed to be performed, and experiencing unpleasant emotions.

\section{Discussion}

To our knowledge, the present project is the first attempt, outside the original Chinese context, to: establish a latent structure of the SMFS, examine whether the factor structure ascertained through EFA fits the data from the second sample and yields a comparable goodness-of-fit index as the original model, and verify whether social media fatigue is associated with Internet addiction. It is also the first study to validate the SMFS on quite large samples of adolescents and young adults.

Our results suggest that the three-factor structure is the optimal solution and corresponds to the theoretical and empirical model of the original SMFS. In the EFA analysis, all three factors accounted for $55.97 \%$ of the variance, in line with the accepted variance of not less than $50 \%$ in the social sciences. ${ }^{58}$ Moreover, the third factor accounted for $11.49 \%$, going well beyond the minimum of $5 \%$ of the 


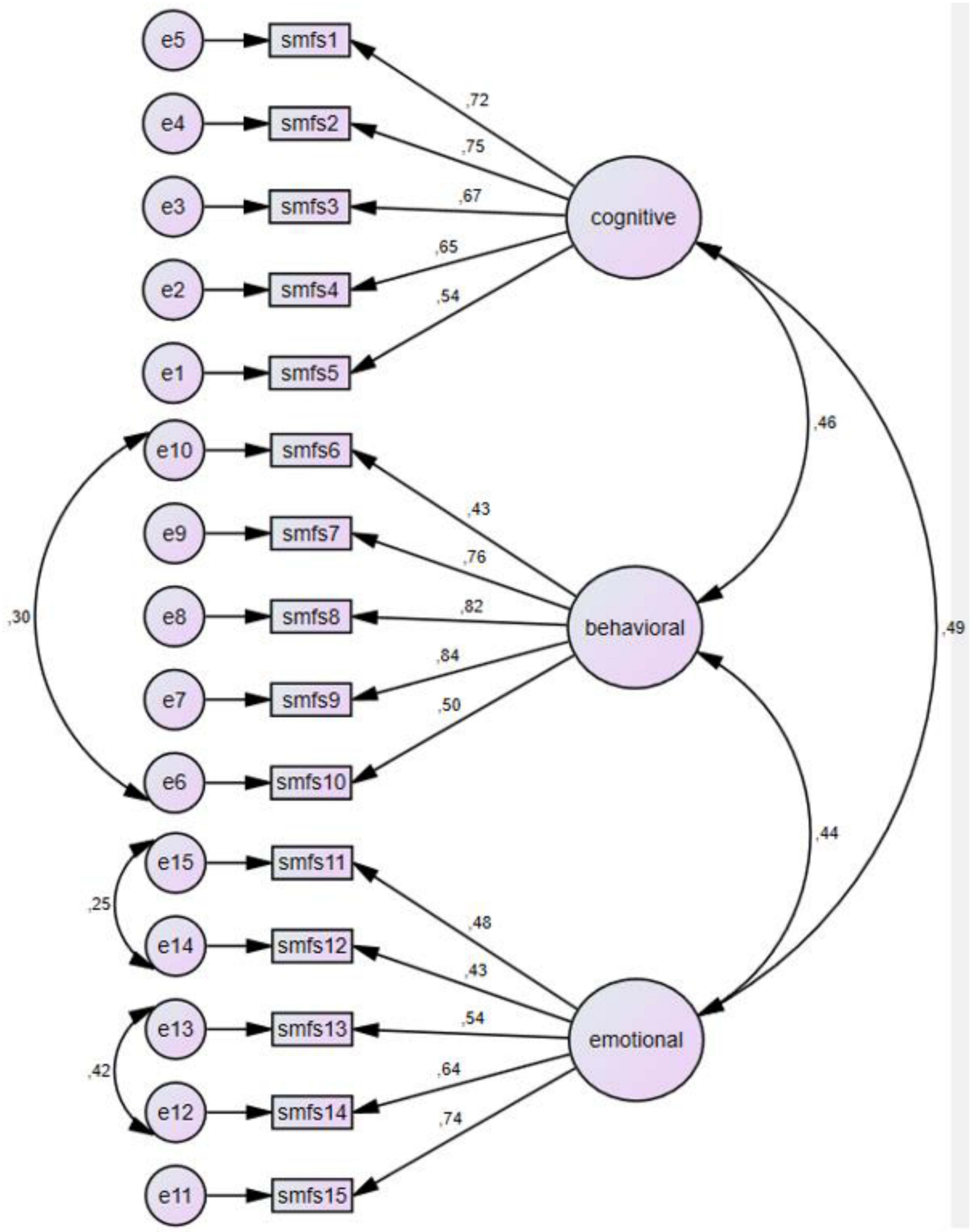

Figure 2 CFA for the SMFS. 
Table 3 Correlations Between Dimensions of SMFS and IAT

\begin{tabular}{|l|c|c|c|c|}
\hline & SMFS_C & SMFS_B & SMFS_E & IAT \\
\hline SMFS_C & I & & & \\
SMFS_B & $0.43^{* * *}$ & $\mathrm{I}$ & & \\
SMFS_E & $0.40^{* * *}$ & $0.40^{* * *}$ & $\mathrm{I}$ & \\
IAT & $0.19^{* * *}$ & $0.38^{* * *}$ & $0.27^{* * *}$ & $\mathrm{I}$ \\
\hline
\end{tabular}

Note: $* * * p<0.001$.

Abbreviations: SMFS_C, cognitive social media fatigue; SMFS_B, behavioral social media fatigue; SMFS_E, emotional social media fatigue; IAT, Internet Addiction Test.

total variance. The results of the reliability analyses also confirm that three dimensions have sufficient reliability, exceeding the level commonly assumed as acceptable (above 0.70). The values of the factor loadings were at an adequate level (between 0.35 and 0.89 ), showing many similarities with the original Chinese version of the SMFS (between 0.40 and 0.72). Although the factor loading of the item smfs 12 had the lowest value among all the items of the SMFS, it can still be considered meaningful and significant since it amounts to about $10 \%$ of the overlapping variance ${ }^{59}$ with four other items of emotional SMF. Moreover, Preacher and MacCallum ${ }^{60}$ postulate that depending on the scientific discipline, large loadings may denote even around 0.30 or 0.4 . Summarizing this part of the study and its overall results, we can say that the Polish version of the SMFS presents satisfactory psychometric properties.

With respect to the CFA, the first analysis provided a marginal fit of the model to the data which, after adding three covariance paths, improved and showed a good fit. The rationale for allowing the correlation of errors lies in the theoretical similarity of the items that belong to the same factors. In case of behavioral fatigue, smfs6 and smfs 10 reflect the reality of the difficulty of coming up with new ideas while updating your own status on social media sites. In case of emotional fatigue, $\operatorname{smfs} 11, \operatorname{smfs} 12$, smfs13, and smfs14 concern annoyance, irritation, and anxiety while using social media.

Finally, all dimensions of SMF correlated positively with Internet addiction, confirming the findings obtained by other researchers. For example, Dol ${ }^{61}$ found that university students who declared feeling fatigue spent more time using social media per day. Lian et $\mathrm{al}^{44}$ observed that fatigue positively correlated with social networking site addiction $(r=0.45)$ among undergraduate students. Excessive Internet use may lead to discomfort and negative emotions related to feeling overwhelmed by information, social interaction overload, and personal or others' expectations.

\section{Limitations}

The present study has some limitations. The research was carried out only among adolescents and young adults. In future studies, it would be valuable to involve other age groups (middle age adults and seniors) to verify the structure of the SMFS among those groups of social media users. Another limitation is related to the disproportionate composition of both samples with respect to sex. Therefore, to assure more solid conclusions, it would be important to enlarge the group of men.

\section{Conclusion}

The present research is the first and a preliminary validation of the Polish version of the Social Media Fatigue Scale. The results of EFA and CFA suggest that it has good psychometric properties and measures the cognitive, behavioral, and emotional dimensions of fatigue related to social networking sites. Moreover, the outcomes show that the SMFS can also be used among adolescents who are largely exposed to the risk of tiredness related to excessive use of the Internet.

With respect to future perspectives, the topic of SMF opens up a wide range of research concerning its potential correlates and determinants. First of all, it is worth verifying whether and how SMF is related to the levels of social activity and the number of close relationships, including the quality of the bonds maintained. Another promising field of investigation would be a study of SMF in the context of both within and beyond the Big Five personality traits. For example, would being a very sensitive person favor an early or more intense experience of SMF? How high would SMF's intensity have to be for people to voluntarily quit social media, remain lonely, or be driven into apathy? In turn, what conditions would have to be met for Internet users to enjoy being inside of social media and not feel overwhelmed by the quantity of information and social networks? Besides a direct association between different factors and SMF, it would also be valuable to examine the effects of possible mediators or moderators on this relationship.

\section{Data Sharing Statement}

The data sets used during the current study are available from the corresponding author. 


\section{Acknowledgment}

The authors thank the study participants who completed the questionnaires.

\section{Author Contributions}

All authors contributed to data analysis, drafting or revising the article, gave final approval of the version to be published, and agree to be accountable for all aspects of the work.

\section{Disclosure}

The authors report no conflicts of interest in this work.

\section{References}

1. Website: datareportal.com. Digital 2021: Global Overview Report; 2021 [updated January 27, 2021]. Available from: https://datarepor tal.com/reports/digital-2021-global-overview-report. Accessed May 19, 2021.

2. Website: statista.com. Share of internet users in Poland from 2019 to 2020, by age group; 2020 [updated December 16, 2020]. Available from: https://www.statista.com/statistics/1015451/poland-internetusers-by-age-group/. Accessed May 19, 2021.

3. Bright LF, Logan K. Is my fear of missing out (FOMO) causing fatigue? Advertising, social media fatigue, and the implications for concumers and brands. INTR. 2018;28(5):1213-1227. doi:10.1108/ IntR-03-2017-0112

4. Liu C, Ma J. Social media addiction and burnout: the mediating roles of envy and social media use anxiety. Curr Psychol. 2018;39 (6):1883-1891. doi:10.1007/s12144-018-9998-0

5. Zhang S, Shen Y, Xin T, Sun H, Wang Y, Zhang X. The development and validation of a social media fatigue scale: from a cognitive-behavioral-emotional perspective. PLoS One. 2021;16(1): e0245464. doi:10.1371/journal.pone.0245464

6. Hanley SM, Watt SE, Coventry W. Taking a break: the effect of taking a vacation from Facebook and Instagram on subjective well-being. PLoS One. 2019;14(6):e0217743. doi:10.1371/journal. pone. 0217743

7. Jorge A. Social media, interrupted: users recounting temporary disconnection on Instagram. Soc Media Soc. 2019;1(2):1-19. doi:10.1177/2056305119881691

8. Hong S, Oh SK. Why people don't use Facebook anymore? An investigation into the relationship between the Big Five personality traits and the motivation to leave Facebook. Front Psychol. 2020;11:1497. doi:10.3389/fpsyg.2020.01497

9. Zhang Y, He W, Peng L. How perceived pressure affects users' social media fatigue behavior: a case on WeChat. J Comput Inform Syst. 2020. doi:10.1080/08874417.2020.1824596

10. Guo Y, Lu Z, Kuang H, Wang C. Information avoidance behawior on social network sites: information irrelevance, overload, and the moderating role of time pressure. Int J Inf Manag. 2020;52. doi:10.1016/j. ijinfomgt.2020.102067

11. Malik A, Dhir A, Kaur P, Johri A. Correlates of social media fatigue and academic performance decrement: a large cross-sectional study. Inf Technol People. 2020;34(2):557-580. doi:10.1108/ITP-06-2019-0289

12. Tugtekin U, Tugtekin EB, Kurt AA, Demir K. Association between fear of missing out, problematic smartphone use, and social networking services fatigue among young adults. Soc Media Soc. 2020;6 (4):1-17. doi:10.1177/2056305120963760
13. Ravindran T, Kuan ACY, Lian DGH. Antecedents and effects of social network fatigue. $J$ Assoc Inf Sci Technol. 2014;65 (11):2306-2320. doi:10.1002/asi.23122

14. Zhang S, Zhao L, Lu Y, Yang J. Do you get tired of socializing? An emppirical explanation of discontiuous usage behavior in social network servises. Inf Manag. 2016;53(7):904-914. doi:10.1016/j. im.2016.03.006

15. Xiao L, Mou J, Huang L. Exploring the antecedents of social network service fatigue: a socio-technical perspective. IMDS. 2019;119 (9):2006-2032. doi:10.1108/IMDS-04-2019-0231

16. Han B. Social media burnout: definition, measurement instrument, and why we care. J Comput Inform Syst. 2018;58(2):122-130. doi:10.1080/08874417.2016.1208064

17. Bright LF, Kleiser SB, Grau SL. Too much Facebook? An exploratory examination of social media fatigue. Comput Hum Behav. 2015;44:148155. doi:10.1016/j.chb.2014.11.048

18. Dhir A, Yossatorn Y, Kaur P, Chen S. Online social media fatigue and psychological wellbeing-A study of compulsive use, fear of missing out, fatigue, anxiety and depression. Int $J$ Inf Manag. 2018;40:141-152. doi:10.1016/j.ijinfomgt.2018.01.012

19. Islam AKMN, Laato S, Talukder S, Sutinen E. Misinformation sharing and social media fatigue during COVID-19: an affordance and cognitive load perspective. Technol Foresast Soc Change. 2020;159:120201. doi:10.1016/j.techfore.2020.120201

20. Lee AR, Son SM, Kim KK. Information and communication technology overload and social networking service fatigue: a stress perspective. Comput Hum Behav. 2016;55:51-61. doi:10.1016/j.chb.2015.08.011

21. Lee E, Lee KY, Sung Y, Song YA. \#DeleteFacebook: antecedents of Facebook fatigue. Cyberpsychol Behav Soc Netw. 2019;22 (6):417-422. doi:10.1089/cyber.2018.0200

22. Xiao L, Mou J. Social media fatigue - technological antecedents and the moderating roles of personality traits: the case of WeChat. Comput Hum Behav. 2019;101:297-310. doi:10.1016/j.chb.2019.08.001

23. Logan K, Bright LF, Grau SL. "Unfriend me, please!": social media fatigue and the theory of rational choice. $J$ Mark Theory Pract. 2018;26(4):357-367. doi:10.1080/10696679.2018.148819

24. Seo Y, Primovic MJ, Jin Y. Overcoming stakeholder social media fatigue: a trialogue approach. J Bus Strategy. 2019;40(6):40-48. doi:10.1108/JBS-04-2019-0071

25. Dai B, Ali A, Wang H. Exploring information avoidance intention of social media users: a cognition-affect-conation perspective. Internet Res. 2020;30(5):1455-1478. doi:10.1108/intr-06-2019-0225

26. Fu S, Li H, Liu Y, Pirkkalainen H, Salo M. Social media overload, exhaustion, and use discontinuance: examining the effects of information overload, system feature overload, and social overload. Inf Process Manag. 2020;57(6):102307. doi:10.1016/j. ipm.2020.102307

27. Matthes J, Karsay K, Schmuck D, Stevic A. "Too much to handle": impact of mobile social networking sites on information overload, depressive symptoms, and well-being. Comput Hum Behav. 2020;105:106217. doi:10.1016/j.chb.2019.106217

28. Hwang MY, Hong JC, Tai KH, Chen JT, Gouldthorp T. The relationship between the online social anxiety, perceived information overload and fatigue, and job engagement of civil servant LINE users. Gov Inf Q. 2020;37(1):101423. doi:10.1016/j.giq.2019.101423

29. Nickerson RS, Adams MJ. Long-term memory for a common object. Cogn Psychol. 1979;11(3):287-307. doi:10.1016/0010-0285(79) 90013-6

30. White KG. Dissociation of short-term forgetting from the passage of time. J Exp Psychol Learn Mem Cogn. 2012;38(1):255-259. doi:10.10137/a0025197

31. Luqman A, Masood A, Weng QD, Ali A, Rasheed MI. Linking excessive SNS use, technological friction, strain, and discontinuance: the moderating role of guilt. Inf Syst Manag. 2020;37(2):94-112. doi: $10.1080 / 10580530.2020 .1732527$ 
32. Pontes HM. Investigating the differential effects of social networking site addiction and Internet gaming disorder on psychological health. J Behav Addict. 2017;6(4):601-610. doi:10.1556/2006.6.2017.075

33. Vanucci A, Flannery KM, McCauley Ohannessian C. Social media use and anxiety in emerging adults. $J$ Affect Disord. 2017;207:163-166. doi:10.1016/j.jad.2016.08.040

34. Whelan E, Islam AKM, Brooks S. Is boredom proneness related to social media overload and fatigue? A stress-outcome approach. Internet Res. 2020;30(3):869-887. doi:10.1108/INTR-03-2019-0112

35. Maier C, Laumer S, Eckhardt A, Weitzel T. When social networking turns to social overload: explaining the stress, emotional exhaustion, and quitting behavior from social network sites' users. Ecis. 2012:1-12.

36. Lee CC, Chou STH, Huang YR. A study on personality and social media fatigue - example of Facebook users. Lecture Notes Info Theory. 2014;2(3):249-253. doi:10.12720/lnit.2.3.249-253

37. Lang A. The limited capacity model of mediated message processing. $J$ Commun. 2006;50(1):46-70. doi:10.1111/j.1460-2466.2000. tb02833.x

38. Lang A. Using the limited capacity model of motivated mediated message processing to design effective cancer communication messages. J Commun. 2006;56(1):57-80. doi:10.1111/j.14602466.2006.00283.x

39. Alavi SS, Maracy MR, Jannatifard F, Eslami M. The effect of psychiatric symptoms on the Internet addiction disorder in Isfahan's University students. J Res Med Sci. 2011;16(6):793-800.

40. Lin SC, Tsai KW, Chen MW, Koo M. Association between fatigue and Internet addiction in female hospital nurses. J Adv Nurs. 2012;69 (2):374-383. doi:10.1111/j.1365-2648.2012.06016.x

41. Bener A, Yildirim E, Torun P, et al. Internet addiction, fatigue, and sleep problems among adolescents students: a large-scale study. Int J Ment Health Addict. 2019;17(4):959-969. doi:10.1007/s11469018-9937-1

42. Duradoni M, Innocenti F, Guazzini A. Well-being and social media: a systematic review of Bergen Addiction Scales. Future Internet. 2020;12(2):24. doi:10.3390/fi12020024

43. Sayeed A, Rahman MH, Hassan MN, et al. Problematic internet use associated with depression, health, and internet-use behaviors among University students of Bangladesh: a cross-sectional study. Child Youth Serv Rev. 2021;120(10):105771. doi:10.1016/j. childyouth.2020.105771

44. Lian SI, Sun HJ, Zhou ZK, Fan CY, Niu GF, Liu QQ. Social networking site addiction and undergraduate students' irrational procrastination: the mediating role of social networking site fatigue and the moderating role of effortful control. PLoS One. 2018;13(12): e0208162. doi:10.1371/journal.pone.0208162

45. O'Keeffe GS, Clarke-Pearson K. Council on Communications and Media. Pediatrics. 2011;127(4):800-804. doi:10.1542/peds.20110054

46. Tomczyk Ł. Evaluation of digital piracy by youths. Future Internet. 2021;13(1):11. doi:10.3390/fi13010011

Psychology Research and Behavior Management
47. Reid D, Weigle P. Social media use among adolescents: benefits and risks. Adolesc Psychiatry. 2014;4(2):73-80. doi:10.2174/ 221067660402140709115810

48. Shapiro LAS, Margolin G. Growing up wired: social networking sites and adolescent psychological development. Clin Child Fam Psychol Rev. 2014;17(1):1-18. doi:10.1007/s10567-013-0135-1

49. Faul F, Erdfelder E, Lang AG, Buchner A. G*Power 3: a flexible statistical power analysis program for the social, behavioral, and biomedical sciences. Behav Res Methods. 2007;39(2):175-191. doi:10.3758/BF03193146

50. Gignac GE, Szodorai ET. Effect size guidelines for individual differences researchers. Pers Individ Dif. 2016;102:74-78. doi:10.1016/j. paid.2016.06.069

51. Schmitt TA, Sass DA. Rotation criteria and hypothesis testing for exploratory factor analysis: implications for factor pattern loadings and interfactor correlations. Educ Psychol Meas. 2011;71(1):95-113. doi: $10.1177 / 0013164410387348$

52. Zhang G, Preacher KJ, Luo S. Bootstrap confidence intervals for ordinary least squares factor loadings and correlations in explorary factor analysis. Multivariate Behav Res. 2010;45(1):104-134. doi:10.1080/002273170903504836

53. Hawi NS, Blachnio A, Przepiorka A. Polish validation of the internet addiction test. Comput Hum Behav. 2015;48:548-553. doi:10.1016/j. chb.2015.01.058

54. Frangos CC, Frangos CC, Sotiropoulos I. A meta-analysis of the reliability of Test. Proc WCE. 2012;1:3-8.

55. Moss TP, Lawson V, White P. The appearance research collaboration. Identification of the underlying factor structure of the Derriford Appearance Scale 24. Peer J. 2015;3:e1070. doi:10.7717/peerj.1070

56. Szcześniak M, Świątek AH, Świątek MA, Rodzeń W. Positive Downstream Indirect Reciprocity Scale (PoDIRS-6): construction and psychometric characteristics. Curr Psychol. 2020. doi:10.1007/ s12144-020-00942-7

57. Worthington RL, Whittaker TA. Scale development research: a content analysis and recommendations for best practices. Couns Psychol. 2006;34(6):806-838. doi:10.1177/0011000006288127

58. Netemeyer RG, Bearden WO, Sharma S. Scaling Procedures: Issues and Applications. Thousand Oaks, CA: Sage Publications; 2003.

59. Makhubela M, Mashegoane S. Establishing factorial validity of the Rosenberg self-esteem scale. In: Laher S, Fynn A, Kramer S, editors. Transforming Research Methods in the Social Sciences: Case Studies from South Africa. London: Sage Publications; 2019:52-68.

60. Preacher KJ, MacCallum RC. Repairing Tom Swift's Electric Factor Analysis Machine. Underst Stat. 2003;2(1):13-43. doi:10.1207/ S15328031US0201 02

61. Dol KS. Fatigue and pain related to internet usage among University students. J Phys Ther Sci. 2016;28(4):1233-1237. doi:10.1589/ jpts. 28.1233

\section{Publish your work in this journal}

Psychology Research and Behavior Management is an international, peer-reviewed, open access journal focusing on the science of psychology and its application in behavior management to develop improved outcomes in the clinical, educational, sports and business arenas. Specific topics covered in the journal include: Neuroscience, memory and decision making; Behavior modification and management; Clinical applications; Business and sports performance management; Social and developmental studies; Animal studies. The manuscript management system is completely online and includes a very quick and fair peer-review system, which is all easy to use. Visit http://www. dovepress.com/testimonials.php to read real quotes from published authors. 